Distribution of Small Molecular Weight Drugs into the Porcine Lens : Studies on Imaging Mass Spectrometry, Partition Coefficients, and Implications in Ocular Pharmacokinetics

\author{
Heilkkinen, Emma M.
}

2019-09

Heilkkinen, E M , Auriola , S , Ranta , V-P , Demarais , N J , Grey , A C , del Amo , E M , Toropainen, E , Vellonen, K-S , Urtti , A \& Ruponen , M 2019 , ' Distribution of Small Molecular Weight Drugs into the Porcine Lens : Studies on Imaging Mass Spectrometry, Partition Coefficients, and Implications in Ocular Pharmacokinetics ', Molecular Pharmaceutics, vol. 16 , no. 9 , pp. 3968-3976 . https://doi.org/10.1021/acs.molpharmaceut.9b00585

http://hdl.handle.net/10138/305523

https://doi.org/10.1021/acs.molpharmaceut.9b00585

cc_by

publishedVersion

Downloaded from Helda, University of Helsinki institutional repository.

This is an electronic reprint of the original article.

This reprint may differ from the original in pagination and typographic detail.

Please cite the original version. 


\title{
Distribution of Small Molecular Weight Drugs into the Porcine Lens: Studies on Imaging Mass Spectrometry, Partition Coefficients, and Implications in Ocular Pharmacokinetics
}

\author{
Emma M. Heikkinen, ${ }^{*} \dagger \odot$ Seppo Auriola, ${ }^{\ddagger}$ Veli-Pekka Ranta, ${ }^{\dagger}$ Nicholas J. Demarais, ${ }^{\S}$ Angus C. Grey,"
}

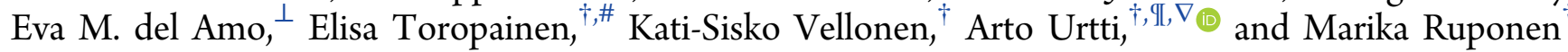

${ }^{\dagger}$ School of Pharmacy, Biopharmacy Department, ${ }^{\star}$ School of Pharmacy, Pharmaceutical Chemistry Department, and " Institute of Clinical Medicine, University of Eastern Finland, Yliopistonranta 1, Kuopio 70211, Finland

${ }^{\S}$ School of Biological Sciences, University of Auckland, Private Bag 92019, Auckland 1142, New Zealand

"University of Auckland, School of Medical Sciences, Department of Physiology, Private Bag 92019, Auckland 1142, New Zealand

${ }^{\perp}$ School of Health Sciences, Division of Pharmacy \& Optometry, University of Manchester, Oxford Road, Manchester M13 9PL, U.K.

${ }^{\mathbb{I}}$ Faculty of Pharmacy, Division of Pharmaceutical Biosciences, University of Helsinki, Viikinkaari, Helsinki 00014, Finland

${ }^{\nabla}$ Institute of Chemistry, Saint Petersburg State University, 26 Universitetskii Prospect, Saint Petersburg 198504, Russia

Supporting Information

ABSTRACT: Lens is the avascular tissue in the eye between the aqueous humor and vitreous. Drug binding to the lens might affect ocular pharmacokinetics, and the binding may also have a pharmacological role in drug-induced cataract and cataract treatment. Drug distribution in the lens has been studied in vitro with many compounds; however, the experimental methods vary, no detailed information on distribution between the lens sublayers exist, and the partition coefficients are reported rarely. Therefore, our objectives were to clarify drug localization in the lens layers and establish

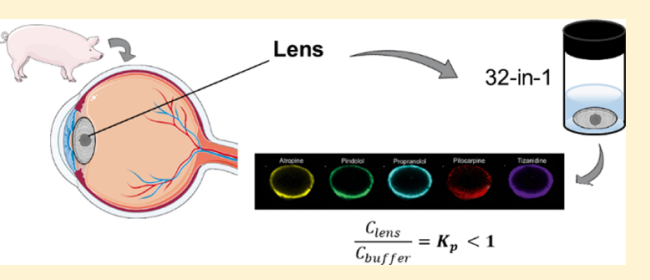
partition coefficients for a wide range of molecules. Furthermore, we aimed to illustrate the effect of lenticular drug binding on overall ocular drug pharmacokinetics. We studied the distribution of 16 drugs and three fluorescent dyes in whole porcine lenses in vitro with imaging mass spectrometry and fluorescence microscopy techniques. Furthermore, we determined lens/buffer partition coefficients with the same experimental setup for 28 drugs with mass spectrometry. Finally, the effect of lenticular binding of drugs on aqueous humor drug exposure was explored with pharmacokinetic simulations. After 4 h, the drugs and the dyes distributed only to the outermost lens layers (capsule and cortex). The lens/buffer partition coefficients for the drugs were low, ranging from 0.05 to 0.8 . On the basis of the pharmacokinetic simulations, a high lens-aqueous humor partition coefficient increases drug exposure in the lens but does not significantly alter the pharmacokinetics in the aqueous humor. To conclude, the lens seems to act mainly as a physical barrier for drug distribution in the eye, and drug binding to the lens affects mainly the drug pharmacokinetics in the lens.

KEYWORDS: lens, drug distribution, ocular, pharmacokinetics, imaging mass spectrometry, MALDI-IMS

\section{INTRODUCTION}

Lens is a transparent, avascular organ positioned in the eye between the aqueous humor and vitreous. The main function of the lens is to change the focal distance of the eye, which ensures the formation of a clear image to the retina with various object distances. The lens consists of the lens capsule, lens anterior epithelial cell monolayer, and lens fiber cells ${ }^{1}$ (Figure S1). The lens capsule surrounds the epithelial and fiber cells of the lens and allows the permeation of even large molecules to the lens. ${ }^{2-4}$ The lens epithelial cell layer lies in the anterior lens and possesses tight junctions. ${ }^{5}$ The fiber cells, named for their long length, are differentiated epithelial cells that have migrated from the anterior lens to the lens equator and further toward the lens center during lens growth. The lens fiber cells can be further divided into loosely structured lens cortex, which consists of the young lens fiber cells, and dense lens nucleus, which consists of the oldest. The main components of the lens are water and proteins, mainly various crystallins, and their concentrations vary between the lens cortex and nucleus, the cortex having higher water and lower protein content than the nucleus. ${ }^{1}$ The lens lipids contain a high level of cholesterol, and they are mostly associated with proteins in the cell membranes. ${ }^{6}$ Thus, the lipid content of lens also increases from the cortex to the nucleus. For a more

Received: May 28, 2019

Revised: July 19, 2019

Accepted: July 26, 2019

Published: July 26, 2019 
detailed, yet introductory, description of the anatomy of the lens, see the Supporting Information.

The role of lens in ocular pharmacology is known to some extent. First, the lens acts as a physical barrier that limits drug entrance from the anterior chamber to the vitreous and vice versa. ${ }^{7}$ Therefore, drug distribution between the anterior and posterior parts of the eye becomes easier in aphakic eyes. ${ }^{8,9}$ Second, drugs may bind to the lens, thereby affecting ocular pharmacokinetics. Third, the lens is the target tissue in potential drug treatments of cataract (i.e., lens opacity). ${ }^{10-12}$ For a pharmacological effect, the drug must diffuse deep enough into the lens. Studies on anticataract drugs have not explored the actual drug distribution or binding in the lens, though the pharmacological effect indicates that at least a low drug concentration in the target site is achieved. On the other hand, drug binding to the lens may also result in cataract formation, for example, with the use of corticosteroids, phenothiazines, and busulfan. ${ }^{13}$

Drug distribution to the isolated lenses has been studied with various compounds, such as pilocarpine, ${ }^{14}$ chloramphenicol, ${ }^{15}$ dexamethasone, ${ }^{15}$ epinephrine, ${ }^{15}$ pilocarpine, ${ }^{15}$ timolol, ${ }^{16}$ and some aldose reductase inhibitors. ${ }^{17}$ Drug distribution between the lens capsule, cortex, and nucleus, however, has not been reported in detail in the literature. One comprehensive study with 13 small molecular weight drugs investigated the in vitro drug partitioning to rabbit lens and linked compound lipophilicity with increased lens-incubate concentration ratio and uptake rate to the lens. ${ }^{18}$ This study also reported concentrations of some drugs in the lens capsule and body (lens epithelium and fiber cells) and concluded that lipophilic drugs can penetrate to the lens body and show higher lens affinity than more polar compounds. In these studies, the experimental methods vary, and only rarely actual lens/buffer partition coefficients were reported.

Drug distribution between the lens capsule and the epithelium-cortex-nucleus has been previously studied with radiolabeled compounds, necessitating the mechanical isolation of different layers of the lens. ${ }^{14}$ Imaging mass spectrometry (IMS) is a novel technique that should enable analysis of various compounds simultaneously in the lens tissue at high spatial resolution $(\approx 10 \mu \mathrm{m})$. $^{19-21}$

In the present work, we aimed to study the localization of various compounds in porcine lens with matrix-assisted laser desorption/ionization IMS (MALDI-IMS) and fluorescence microscopy. Furthermore, we determined the lens/buffer partition coefficients $\left(K_{\mathrm{p}}\right)$ for 28 drugs in the isolated porcine lens with liquid chromatography-mass spectrometry (LCMS/MS). Finally, we illustrated the pharmacokinetic role of lenticular drug distribution with pharmacokinetic simulations.

\section{MATERIALS AND METHODS}

2.1. Tissue Isolation. Enucleated porcine eyes were received from a local slaughterhouse and transported to the laboratory in cooled $25 \mathrm{mM}$ phosphate-buffered saline (PBS) within $6 \mathrm{~h}$ after enucleation. Extraocular tissues were removed from the isolated eyeballs. Then, the eye was cut open from limbus and the lens was collected carefully with a spatula. The lenses were weighed and immediately used in the experiments.

2.2. Extent of Drug Distribution into the Isolated Porcine Lens. 2.2.1. Preparation of the Cassette Mix. Drug distribution to the lenses was determined by using a mixture of 32 compounds: the compounds and their chemical descriptors, vendors, and solvents for stock solutions are listed in ref. ${ }^{22}$
Lornoxicam was excluded from the mix. Stock solutions [0.5$10 \mathrm{mg} / \mathrm{mL}$ in PBS or dimethyl sulfoxide (DMSO)] were combined and diluted further with Hanks' balanced salt solution (HBSS)-HEPES (25 mM) ( $\mathrm{pH} 7.4)$. Octanol-water distribution coefficients of the compounds $\left(\log D_{7.4}\right)$ and polar surface areas (PSAs) were previously estimated in silico from compound structures with ACD/Labs-software (v12, Advanced Chemistry Development, Inc., Canada). ${ }^{22}$

2.2.2. Drug Distribution into the Lens: Studies for MALDIIMS. 2.2.2.1. Incubation. To determine distribution of cassette mix drugs in the lens, the isolated porcine lenses $(n=10)$ were incubated in $1200 \mu \mathrm{L}$ of prewarmed drug solution in capped flat-bottomed polystyrene vials sealed with parafilm at $+35^{\circ} \mathrm{C}$ in a horizontal shaker (Heidolph incubator 1000, Heidolph Elektro $\mathrm{GmbH} \&$ Co., Germany) at $150 \mathrm{rpm}$ shaking. After 4 $h$, the buffer was removed from the vials and the lenses were rinsed with HBSS-HEPES and gently blotted dry. The lenses were weighed, snap-frozen with liquid nitrogen in flatbottomed plastic tubes, and then stored at $-80{ }^{\circ} \mathrm{C}$ until sample preparation and analysis with MALDI-IMS. For the experiment, a smaller set of 16 drugs was used. The final incubation mixture of drugs consisted of acetazolamide, ampicillin, atenolol, atropine, betaxolol, carteolol, ciproflocaxin, ketorolac, lincomycin, nadolol, pilocarpine, pindolol, and propranolol, each at $10 \mu \mathrm{g} / \mathrm{mL}$. Also, aztreonam, methazolamide, and tizanidine were included at $100 \mu \mathrm{g} / \mathrm{mL}$. Final DMSO concentration of the solution was $2 \%$.

2.2.2.2. Tissue Preparation. The frozen lenses were mounted onto a chuck using an optimal cutting temperature compound (Sakura Finetek, CA, USA) on the equatorial side. The lenses were sectioned to $10 \mu \mathrm{m}$ thickness at $-20{ }^{\circ} \mathrm{C}$ on a Leica CM Cryostat (S3050, Leica Microsystems GmbH, Germany), equipped with a FEATHER Microtome C35 blade (pfm medical, UK), and collected on a cryofilm (3C16UF, SECTION-LAB Co. Ltd. Yokohama, Japan) by modified Kawamoto method. ${ }^{23}$ The film was subsequently mounted onto a microscopy glass slide using a double-sided copper tape. The slides were washed twice with $50 \mathrm{mM}$ ammonium formate for $30 \mathrm{~s}$ and dried in a vacuum desiccator. The sample slides contained three lens sections from the drug mixture experiment and three blanks. To quantify the amount of each compound present in incubated lenses, $1 \mu \mathrm{L}$ of standard mixture was added on one of the blank lenses using concentrations of $0.1,0.5,2$, and $10 \mu \mathrm{g} / \mathrm{mL}$. First, the internal standard solution of atropine- $d_{5}(200 \mathrm{ng} / \mathrm{mL}$ in $50 \%$ acetonitrile) was sprayed to obtain a $10.2 \mathrm{ng} / \mathrm{cm}^{2}$ corresponding to approximately $10 \mu \mathrm{g} / \mathrm{g}$ in the tissue. Matrix $\alpha$-cyano-4hydroxycinnamic acid $(7 \mathrm{mg} / \mathrm{mL}$ in $50 \%$ acetonitrile containing $1 \%$ trifluoro acetic acid) (Sigma-Aldrich, St. Louis, MO, USA) was added via spray deposition using 10 passes, flow rate of $100 \mu \mathrm{L} / \mathrm{min}$, temperature of $77^{\circ} \mathrm{C}$, track spacing of $2.5 \mathrm{~mm}$, and velocity of $1300 \mathrm{~mm} / \mathrm{min}$ (HTX Industries, TM-Sprayer, NC, USA).

2.2.2.3. Fourier Transform-Ion Cyclotron Resonance-IMS. MALDI Fourier transform-ion cyclotron resonance (FT-ICR)IMS was performed using a Bruker 7T solariX XR mass spectrometer (Bruker Daltonics, Germany) at a $75 \mu \mathrm{m}$ spatial resolution. Spectra were collected in positive ion mode in mass-to-charge ratio $(\mathrm{m} / z)$ range of $100-1000$, and the resolution was 61000 at $\mathrm{m} / z$ 307. The compounds and $\mathrm{m} / z$ values $\left([\mathrm{M}+\mathrm{H}]^{+}\right)$used were as follows: atropine 290.1751, propranolol 260.1645, atenolol 267.1703, carteolol 293.18601860, pilocarpine 209.1285, methazolamide 
237.0111, pindolol 249.1598, tizanidine 254.0262, nadolol 310.2013, lincomycin 407.2210, and fluconazole 307.1113.

2.2.2.4. Analysis of FT-ICR Data and Image Processing. FT-ICR data were read into Fleximaging 4.1 software (Bruker Daltonics, Germany) and normalized against the $d_{5}$-atropine internal standard signal at $m / z 295$. The drugs were identified as their $[\mathrm{M}+\mathrm{H}]^{+}$ions. Peak finding was performed using mass error less than $2.5 \mathrm{ppm}$. MALDI images for each detected compound were plotted using a $0.01 \mu$ mass window. From the images of the individual drugs, the intensity profiles across a 30 pixel $(2.25 \mathrm{~mm})$ wide area in anterior-posterior axis were plotted with ImageJ software (National Institutes of Health, USA).

2.2.3. Partition Coefficients for Cassette Mix Drugs. 2.2.3.1. Incubation. To determine the $K_{\mathrm{p}}$ values, the distribution study was conducted as described in Section 2.2.2, but the incubation volume $(300 \mu \mathrm{L})$, the number of drugs, and the drug concentrations in the cassette mix were different. For this experiment, the whole mix of 32 drugs was used. The test concentrations of the drugs in the cassette mix were 1 and $10 \mu \mathrm{g} / \mathrm{mL}: 1 \mu \mathrm{g} / \mathrm{mL}$ was used for all compounds except aztreonam, bromfenac, dexamethasone, diclofenac, fluorometholone, indomethacin, levocabastine, methazolamide, prednisolone, quinidine, and tizanidine. For these compounds, a concentration of $10 \mu \mathrm{g} / \mathrm{mL}$ was used. Two different drug concentrations were used to ensure reliable quantitation in the LC-MS/MS analyses. The DMSO concentration of the final mixture was $0.9 \%$. The lenses were stored at $-80{ }^{\circ} \mathrm{C}$ until sample preparation and analysis with LC-MS/MS.

2.2.3.2. Sample Preparation for LC-MS/MS. The lenses were homogenized first with plastic homogenization pestles and then with ULTRA-TURRAX (model T8, IKA-Werke $\mathrm{GmbH} \& \mathrm{Co}$. KG, Germany) in HBSS-HEPES ( $1 \mathrm{~g}$ lens +9 $\mathrm{mL}$ buffer $)$. Lens homogenate $(200 \mu \mathrm{L})$ was mixed with 400 $\mu \mathrm{L}$ of methanol (Chromasolv LC-MS Ultra, Honeywell Riedel-de Haën, NC, USA), containing internal standards atenolol- $d_{7}$ (Toronto Research Chemicals Inc., Canada), atropine- $d_{5}$ (Toronto Research Chemicals Inc., Canada), fluconazole- $d_{4}$ (Toronto Research Chemicals Inc., Canada), and lincomycin- $d_{3}$ (Toronto Research Chemicals Inc., Canada) at $24.4 \mathrm{ng} / \mathrm{mL}$ each and vortexed. The samples were centrifuged at $16060 \mathrm{~g}$ for $10 \mathrm{~min}$ at $+4{ }^{\circ} \mathrm{C}$ and their supernatants were collected and stored at $-20{ }^{\circ} \mathrm{C}$. On the day of LC-MS run, the sample supernatants $(300 \mu \mathrm{L})$ were filtered through a Captiva ND lipid-plate (Agilent Technologies, CA, USA) to a Captiva 96 deep well collection plate (Agilent Technologies, CA, USA) by centrifugation at $1560 \mathrm{~g}$ for $40 \mathrm{~min}$ at $+4{ }^{\circ} \mathrm{C}$. Duplicate standards (eight levels, 5-1000 $\mathrm{ng} / \mathrm{mL}$ ) and triplicate quality control (QC) samples (50, 250 and $1000 \mathrm{ng} / \mathrm{mL}$ ) were prepared from cassette mix working solutions in a similarly diluted lens homogenates as the actual samples.

2.2.3.3. LC-MS/MS Analysis. For detailed description of the LC-MS/MS acquisition method, see ref 22. For the LC-MS/ MS analyses, the lens samples were run in two separate analysis batches with independent standards and QC samples. The resulting data were analyzed with Agilent MassHunter Quantitative Analysis software (vB.09.00, build 9.0.647.0, Agilent Technologies, CA, USA). Internal standards were used in the calculations. The calibration curves were calculated from duplicate standard series, one of which was run in the beginning and the other at the end of each analysis. Quadratic fitting with $1 / x$ weighing was used. The criteria for calibration curve and QC acceptance were $80-120 \%$ accuracy of mean for $\geq 66.7 \%$ of calibration levels and at LLOQ 80-120\% accuracy for $\geq 66.7 \%$ of individual QCs and QC levels and $<20 \%$ coefficient of variation for $\geq 66.7 \%$ of QC levels. The selectivity criterion of $\geq 3$-fold response ratio of LLOQ to matrix-based blank sample was employed.

2.2.3.4. Calculation of Lens/Buffer Partition Coefficients $\left(K_{p}\right)$. From the LC-MS/MS results, $K_{\mathrm{p}}$ values were first calculated using the total lens volume (eq 1 )

$$
K_{\mathrm{p}}=\frac{C_{\text {lens,4h }}}{C_{\text {inc, } 4 \mathrm{~h}}}=\frac{m_{\text {lens }, 4 \mathrm{~h}} / V_{\text {lens }}}{C_{\text {inc, init }}-m_{\text {lens }, 4 \mathrm{~h}} / V_{\text {inc }}}
$$

where $C_{\text {lens, } 4 \mathrm{~h}}$ is the drug concentration in the lens at $4 \mathrm{~h}(\mathrm{ng} /$ $\mathrm{mL}), C_{\text {inc, } 4 \mathrm{~h}}$ is the drug concentration in the incubate at $4 \mathrm{~h}$ $(\mathrm{ng} / \mathrm{mL}), m_{\text {lens,4h }}$ is the drug amount in the lens at $4 \mathrm{~h}(\mathrm{ng})$, $V_{\text {lens }}$ is the lens volume $(\mathrm{mL})$ (calculated with the actual lens mass and lens density of $\left.1.183 \mathrm{~g} / \mathrm{mL}^{24}\right), C_{\text {inc,init }}$ is the initial drug concentration in the incubate $(\mathrm{ng} / \mathrm{mL})$, and $V_{\text {inc }}$ is the volume of the buffer $(\mathrm{mL})$.

The drug concentration in the incubate at $4 \mathrm{~h}$ was calculated by using the initial drug concentration in the buffer $\left(C_{\text {inc,init }}\right)$, buffer volume $\left(V_{\text {inc }}\right)$, and drug amount in the lens at $4 \mathrm{~h}$ $\left(m_{\text {lens,4h }}\right)$ instead of the measured concentration in the incubate because the concentration in the incubate showed very little decrease $(<5-10 \%)$ during the incubation. $K_{\mathrm{p}}$ values were also calculated with the actual lens volume into which the compounds distribute based on the MALDI IMS data (true distribution volume instead of the total lens volume) (see the Supporting Information). The same approximation for the volume of distribution was assumed for all cassette mix compounds.

2.3. Distribution Patterns of Fluorescent Dyes within the Lens. 2.3.1. Fluorescent Dyes. The distribution patterns of the compounds in the porcine lenses were studied with three fluorescent dyes with varying lipophilicities. Rhodamine$\mathrm{B}$ and fluorescein sodium (Sigma-Aldrich) were dissolved into HBSS-HEPES ( $\mathrm{pH} 7.4$ ) at $1 \mathrm{mg} / \mathrm{mL}$ concentration. Rhodamine-123 (Sigma-Aldrich) was first dissolved into ethanol (10 $\mathrm{mg} / \mathrm{mL}$ ) and then diluted in HBSS-HEPES ( $\mathrm{pH} 7.4)$ to reach $0.1 \mathrm{mg} / \mathrm{mL}$ (final ethanol concentration was $1 \%$ ).

2.3.2. Incubation. The distribution studies with fluorescent dyes were conducted separately for each dye, as described in Section 2.2.2, with an incubation volume of $1200 \mu \mathrm{L}$. After incubation, the lenses were rinsed, blotted dry, frozen in an optimal cutting temperature compound (Sakura Finetek, SA, USA) in plastic tubes with chilled 2-propanol, and stored at $-20{ }^{\circ} \mathrm{C}$ until cryosectioning.

2.3.3. Fluorescence Microscopy. The lenses were sectioned to a thickness of $10 \mu \mathrm{m}$ at $-20{ }^{\circ} \mathrm{C}$ with Leica CM cryostat (Leica 3050S, Leica Microsystems GmbH, Germany) equipped with a FEATHER Microtome C35 blade (pfm medical, UK) and collected on a SuperFrost Plus adhesion microscope slide (Thermo Fisher Scientific, MA, USA). The tissue slices were imaged with a fluorescence microscope (Zeiss Axio Imager M2, Carl Zeiss Microscopy GmbH, Germany) equipped with filters $470 \mathrm{~nm}$ (65HEAF488) and $590 \mathrm{~nm}$ (64HEmPlum) filters and Axiocam MRm camera (Carl Zeiss Microscopy GmbH, Germany) using a 2.5-fold magnification. Exposure times were $600 \mathrm{~ms}$ for fluorescein sodium, $600 \mathrm{~ms}$ for rhodamine-B, and $1 \mathrm{~s}$ for rhodamine- 123 . Individual images of the lens sections were merged together manually with GNU image processing software (v2.10.6). 
2.4. Pharmacokinetic Simulations. 2.4.1. Model Structure and Parameters. A pharmacokinetic simulation model for rabbit was built for topical timolol instillation based on the model of Ranta et al. ${ }^{25}$ The lens compartment, separate from the reservoir compartment, was added to the model (Figure 1).

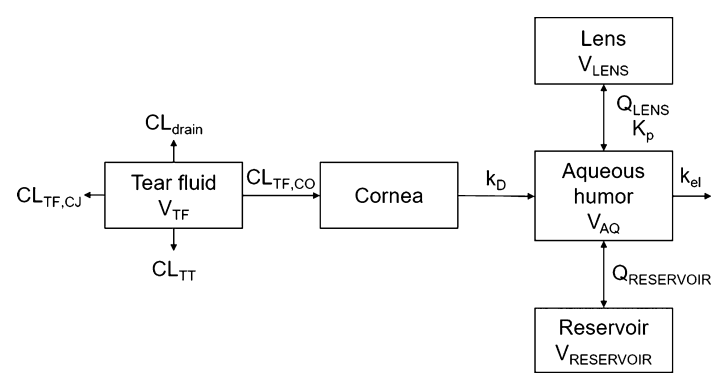

Figure 1. Simulation model for timolol distribution to the lens after topical dosing, extended from ref $25 . V_{\mathrm{TF}}$, tear fluid volume; $\mathrm{CL}_{\mathrm{TF}, \mathrm{C} J}$, clearance from tear fluid by conjunctival absorption; $\mathrm{CL}_{\mathrm{TT}}$, clearance from tear fluid by tear turnover; $\mathrm{CL}_{\text {drain }}$, clearance from tear fluid by drainage of the instilled solution; $\mathrm{CL}_{\mathrm{TF}, \mathrm{CO}}$, clearance from tear fluid by corneal absorption; $k_{\mathrm{D}}$, corneal desorption rate constant; $V_{\mathrm{AQ}}$, aqueous humor volume; $k_{\mathrm{e}}$, elimination rate constant; $Q_{\text {RESERVOIR }}$ distribution clearance between aqueous humor and reservoir; $V_{\text {RESERVOIR }}$ reservoir volume; $K_{\mathrm{p}}$, lens/buffer partition coefficient; $Q_{\text {LENS }}$, distribution clearance between aqueous humor and the lens; $V_{\text {LENS }}$, lens volume.

The unknown parameter values for timolol distribution clearance between aqueous humor and lens $\left(Q_{\text {LENS }}\right), K_{\mathrm{p}}$, and clearance from tear fluid to cornea $\left(\mathrm{CL}_{\mathrm{TF}, \mathrm{CO}}\right)$ were obtained by adjusting the values manually until the simulated concentration in the lens matched with in vivo data on timolol distribution to the rabbit lens. ${ }^{26}$ Finally, the distribution clearance between the aqueous humor and the reservoir $\left(Q_{\text {RESERVOIR }}\right)$ and the volume of the reservoir $\left(V_{\text {RESERVOIR }}\right)$ were adjusted to match the corresponding parameters in the original model. For details of the model building and parameters, see the Supporting Information. STELLA software (v8.1.1, isee systems) was used to construct the simulation model.

2.4.2. Simulations. Kinetic simulations were carried out to estimate how drug partitioning into the lens might affect ocular pharmacokinetics after single and multiple doses of eye drops. In the simulations, a single and repeated dosing (every 24 and $8 \mathrm{~h})$ of $125 \mu \mathrm{g}$ of timolol $(0.5 \%, 25 \mu \mathrm{L})$ were used. The run time was 7 days and the delta time was $0.5 \mathrm{~min}$. Simulations were run with $K_{\mathrm{p}}$ values of $0.35,1.05$ (3-fold increase), and 3.5 (10-fold increase) to see the potential impact of lens partitioning on ocular pharmacokinetics.

\section{RESULTS}

3.1. Lens Integrity. The lens should retain its integrity during the incubation with drugs. Therefore, the integrity was evaluated by monitoring the lens mass (Figure S2) and appearance at different times. During $4 \mathrm{~h}$ of incubation, the mass was retained at $469 \pm 55.8 \mathrm{mg}$, and no change in the lens mass was observed. Furthermore, the lenses did not show damage or swelling during the experiments. However, in a preliminary experiment, a clear decrease in the lens mass was seen at longer incubation times. The remaining mass, compared to the original, was $81.3 \pm 11.0 \%(n=2)$ at $12 \mathrm{~h}$ and $67.2 \pm 16.8 \%(n=10)$ at $24 \mathrm{~h}$ of incubation (Figure S2). Furthermore, after $4 \mathrm{~h}$, the lens capsule and cortex began swelling and came off by $12 \mathrm{~h}$. For this reason, longer incubations were not feasible and incubations of $4 \mathrm{~h}$ were used in the experiments.

3.2. Drug and Dye Distribution Patterns in the Lens. The distribution patterns of the cassette mix drugs in the porcine lens were evaluated using MALDI-IMS. Eleven (atenolol, atropine, carteolol, fluconazole, lincomycin, methazolamide, nadolol, pilocarpine, pindolol, propranolol, and tizanidine) out of the 16 compounds gave acceptable signals when standard solution was loaded on a blank lens section (Figure S3). When the drug amount in the pipetted standard solutions exceeded $2 \mathrm{ng}$ per spot, the internal standard atropine- $d_{5}$ signal was suppressed (Figure S4).

In the lens samples, atropine, pilocarpine, pindolol, propranolol, and tizanidine (in silico predicted $\log D_{7.4}$ range from -1.09 to 2.04 ) showed distribution only in the capsule and cortex of the lens but not in the nucleus (Figure 2). The posterior rim showed 1.2- to 2-fold higher intensities than the anterior rim (Figure 3). In general, the signal intensity-

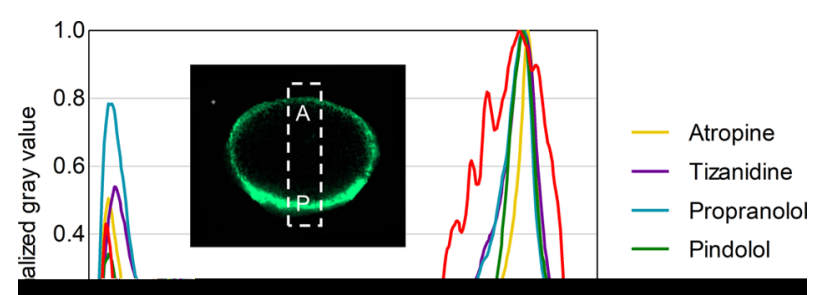

Figure 3. Relative drug distribution along the anterior-posterior axis in a porcine lens after $4 \mathrm{~h}$ in vitro incubation.

distance profiles for the drugs were similar. For aztreonam, lincomycin, methazolamide, and nadolol, the signals at their $\mathrm{m} / z$ were detected in the drug-incubated lens sample, but also in the blank lenses. This indicates that the signals originated from some endogenous compounds with the same $\mathrm{m} / z$ within our experimental error. These compounds were therefore excluded from further analysis. In the average spectrum taken from the lens posterior surface (thickness $1 \mathrm{~mm}$ ), the intensity measured for $d_{5}$-atropine was 850 and for atropine 290 (Figure

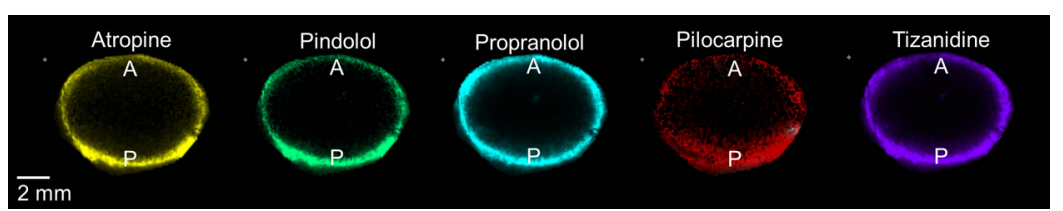

Figure 2. Drug distribution in a porcine lens after $4 \mathrm{~h}$ in vitro incubation. $m / z$ signals across a lens section were obtained with MALDI-IMS and normalized to internal standard atropine- $d_{5}$ signal. $\mathrm{A}=$ anterior lens pole, $\mathrm{P}=$ posterior lens pole. 




